\title{
Pendular Alignment of Paramagnetic Molecules in Uniform Magnetic Fields
}

\author{
Alkwin Slenczka, Bretislav Friedrich, and Dudley Herschbach \\ Department of Chemistry, Hartard University, 12 Oxford Street, Cambridge, Massachusetts 02138
}

(Received 1 November 1993)

\begin{abstract}
For paramagnetic molecules subjected to a uniform magnetic field, the Zeeman interaction produces widely tunable, aligned pendular states in which the molecular magnetic dipole librates about the field direction. We demonstrate such alignment by observing spectroscopic transitions at $\approx 1 \mathrm{~T}$ involving the $A^{3} \Pi_{1}$ excited state of $\mathrm{ICl}$, and extract characteristic pendular eigenproperties that arise from the hybridization of field-free rotor states. Unlike the electric analog, magnetic pendular states can be created for nonpolar molecules and molecular ions.
\end{abstract}

PACS numbers: $32.60 .+i, 35.20 . B m$

It has been recognized [1,2] and demonstrated [1,3-8] that strong uniform electric fields can hybridize low rotational states of polar molecules and thus create sui generis pendular states in which the molecular axis is confined to librate over a limited angular range about the field direction. It has also been recognized [9] that such a hybridization can be brought about for linear Hund's case $(a)$ or $(c)$ molecules, by the interaction of their body-fixed electronic magnetic moments with a uniform magnetic field. In this Letter we present a prototype spectroscopic experiment to demonstrate the magnetic pendular states and their eigenproperties.

In addition to its role in producing oriented or aligned molecules for studies of collision stereodynamics [10], pendular hybridization can be utilized to advantage in spectroscopy. This includes determining molecular parameters $[4,5,8]$, providing intermediate states with widely tunable energies $[2,4,5,9]$, and making accessible states otherwise forbidden by selection rules [4,5,8,11]. Magnetic hybridization offers complementary chemical scope to the electric version by virtue of its applicability to nonpolar paramagnetic molecules and ions.

Figure 1 shows our experimental arrangement. We create magnetic pendular states of $\operatorname{ICl}\left(A^{3} \Pi_{1}\right)$ by sending a supersonic molecular beam of $\mathrm{ICl}\left(X^{1} \Sigma_{0}^{+}\right)$into a uniform magnetic field between the pole pieces of a permanent magnet and pumping a fraction of the molecules to the $A^{3} \Pi_{1}$ excited electronic state by a laser. By scanning the laser wavelength within the $A^{3} \Pi_{1}-X^{1} \Sigma_{0}$ band and measuring the induced fluorescence we simultaneously probe the pendular states produced. The molecular beam contains about $30 \%$ of $\mathrm{ICl}$ seeded in $\mathrm{Ar}$ and is formed by expanding the gas mixture at 50 torr and 310 $\mathrm{K}$ through a glass nozzle $300 \mu \mathrm{m}$ in diameter. The unskimmed cold beam is then passed between a pair of NdFeB magnetic disks (IBS magnet; $25 \mathrm{~mm}$ in diameter and $7 \mathrm{~mm}$ thick, remanence of $1.25 \mathrm{~T}$ ) whose adjustable distance is used to define the magnetic field strength $\mathcal{H}$; this can be varied up to $0.8 \mathrm{~T}$, leaving a gap of about 5 $\mathrm{mm}$. The molecular beam within the gap is illuminated at right angles by the beam of a single-mode tunable ring dye laser (Coherent 699-21); the collected fluorescence is space filtered [12], fed into a fiber bundle (Oriel), and detected by a photomultiplier tube (Hamamatsu 943-02). The transitions were measured within the $v^{\prime}=19-v^{\prime \prime}$ $=0$ vibrational band of the $A^{3} \Pi_{1}-X^{1} \Sigma_{0}^{+}$system (large Franck-Condon factor, little overlap by lines from $\mathrm{I}_{2}$ contamination in the beam [8]) and their hyperfine structure was largely resolved (Doppler linewidth of $\approx 100 \mathrm{MHz}$ ). Figure 2 shows the fluorescence spectra at $\mathscr{H}=0$ and at $\mathscr{H}=0.78 \mathrm{~T}$ excited with parallel and perpendicular linear polarization (with respect to $\mathscr{H}$ ) of the laser. The observed dramatic alterations of the spectra with field strength are due to hybridization and lifting of the selection rules for changes in the total angular momentum quantum number, $J$. The two high-field spectra differ due to the change in the $\Delta M$ selection rule for parallel

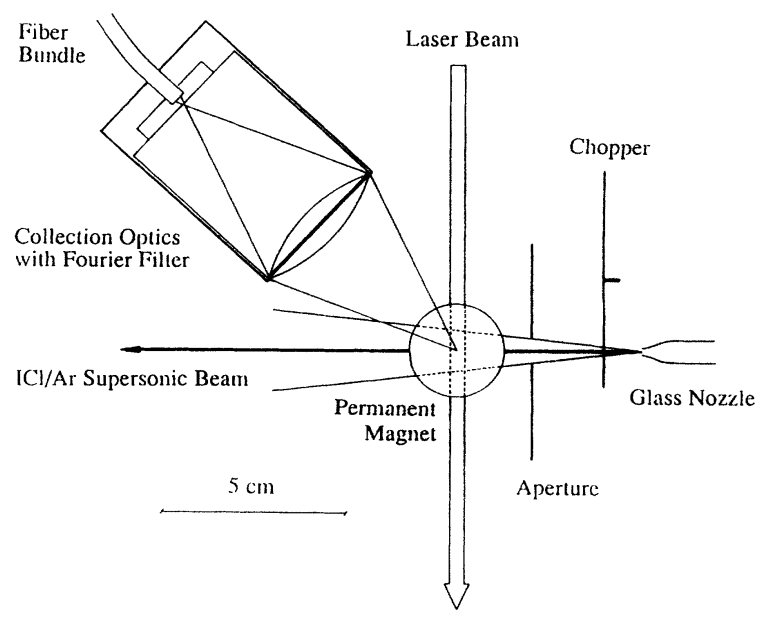

FIG. 1. Experimental setup. The unskimmed supersonic beam of $\mathrm{ICl}$ seeded in $\mathrm{Ar}\left(T_{\text {rot }} \approx 10 \mathrm{~K}\right)$ is passed through a gap between a pair of NdFeB magnetic disks whose adjustable distance is used to define the magnetic field strength of up to 0.8 T. The molecular beam within the gap is illuminated at right angles by the focused beam $\left(6 \mathrm{~W} / \mathrm{cm}^{2}\right)$ of a tunable ring dye laser; the collected fluorescence is space filtered, fed into a fiber bundle, and detected by a photomultiplier tube. Computer controlled lock-in amplification is used to enhance the signal-tobackground ratio. 


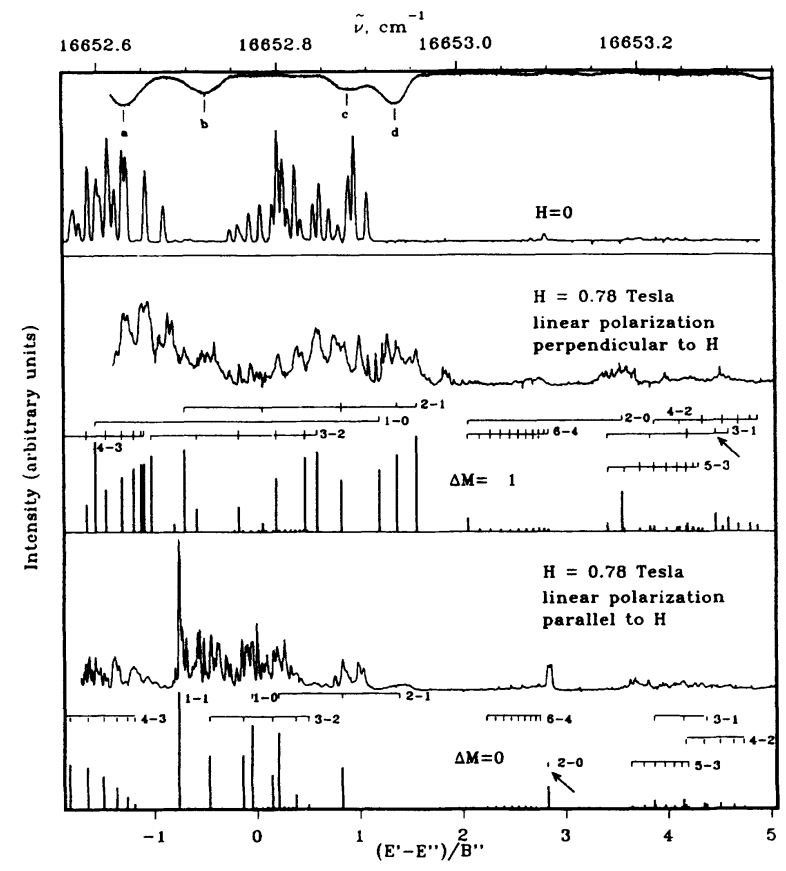

FIG. 2. Observed and calculated laser-induced fluorescence spectra from the $v^{\prime}=19-v^{\prime \prime}=0$ vibrational band of the $A^{3} \Pi_{1}$ $X^{1} \Sigma_{0}^{+}$system of $I^{35} \mathrm{Cl}$ seeded in a supersonic molecular beam of Ar. Upper panel: Field-free spectrum, together with four absorption bands of $I_{2}$ used to calibrate the frequency scale; these occur at $16652+\Delta \tilde{v}$, with $\Delta \tilde{v}=0.6312,0.7232,0.8847$, and 0.9409 (in $\mathrm{cm}^{-1}$ ) for $a, b, c$, and $d$ [13], respectively. Middle panel: Spectrum at field strength $0.78 \mathrm{~T}$, for linear polarization of laser perpendicular to field. Calculated stick spectra (see text) are sorted into bands labeled by $\tilde{J}^{\prime}-J^{\prime \prime}$; within each such band, the lines (from left to right) arise from $M^{\prime}=J^{\prime \prime}+1$ to $-J^{\prime \prime}-1$ for $\Delta \Omega \equiv \Omega^{\prime}-\Omega^{\prime \prime}=+1$; the lower and upper bars indicate $\Delta M \equiv M^{\prime}-M^{\prime \prime}=+1$ and -1 , respectively. Lower panel: Spectrum at $0.78 \mathrm{~T}$, for polarization parallel to field. Within each $\tilde{J}^{\prime}-J^{\prime \prime}$ band $M^{\prime \prime}=M^{\prime}=J^{\prime \prime}, \ldots,-J^{\prime \prime}$ from left to right for $\Delta \Omega=+1$. Note that each perpendicular as well as parallel transition has a twin; see text. Within the field, $\omega^{\prime \prime}=0$ and $\omega^{\prime}=5.82$; see Table 1 . The specimen transitions used in Table II are indicated by arrows.

and perpendicular polarization of the laser.

For a rigid linear molecule in a uniform magnetic field $\mathcal{H}$ the Schrödinger equation is

$$
\left(\mathrm{J}^{2}-\Omega-\omega \cos \theta\right)|\tilde{J}, \Omega, M ; \omega\rangle=E|\tilde{J}, \Omega, M ; \omega\rangle
$$

with $\mathbf{J}^{2}$ the square of the total angular momentum (rotational+electronic, but excluding nuclear spin), $J_{z}$ $=\Omega$ the projection of the electronic angular momentum on the molecular figure axis $z, \theta$ the angle between $z$ and $\mathcal{H}$, and $\omega \equiv \mu \mathscr{H} / B$ the dimensionless parameter which measures the potential energy of the magnetic dipole $\mu$ in units of the rotational constant, $B$. In Hund's case $(a)$ the modulus of the body-fixed magnetic dipole moment endowed by the electronic angular momentum is $\mu$ $\equiv\left(g_{L} \Lambda+g_{S} \Sigma\right) \mu_{B}$ where $\Lambda$ and $\Sigma$ are projections of the or- bital and spin electronic angular momenta $\mathbf{L}$ and $\mathbf{S}$ on the $z$ axis, $g_{L}$ and $g_{S}$ are the respective gyromagnetic ratios $\left(g_{L}=1, g_{S} \approx 2.00229\right)$, and $\mu_{B}$ is the Bohr magneton. The Schrödinger equation (1) becomes equivalent to that for the electric case (leading to electric pendular states) merely by replacing $\mu \mathscr{H}$ by the product of the electric dipole moment and electric field strength. Eigenproperties as functions of $\omega$ are readily determined by expanding the wave functions in terms of the field-free solutions,

$$
|\tilde{J}, \Omega, M ; \omega\rangle=\sum_{J} a_{J \Omega M}(\omega)|J, \Omega, M\rangle
$$

to obtain algebraic equations for the Fourier coefficients $a_{J \Omega M}(\omega)$; these can be solved with arbitrary accuracy by standard methods [5]. The eigenfunctions of Eq. (2) are thus linear superpositions or hybrids of the field-free symmetric top wave functions $|J, \Omega, M\rangle$ for fixed values of the good quantum numbers $\Omega$ and $M$; the range of $J$ involved increases with the $\omega$ parameter. The eigenstates are labeled by $\tilde{J},|\Omega|, M$ where $\tilde{J}$ denotes the nominal value that pertains to the field-free rotational state which adiabatically correlates with the high-field state, $\mid \tilde{J}, \Omega, M ; \omega$ $\rightarrow 0\rangle \rightarrow|J, \Omega, M\rangle$, cf. the correlation diagram in Ref. [9]. In the high field limit, $\omega \rightarrow \infty$, the hybrids correlate with harmonic librator states whose spacing corresponds to the same harmonic frequency. This limit is attained for states with $\tilde{J} \leq 1$ at about $\omega=5$ and at about $\omega=100$ for $\tilde{J}=5$. However, for, e.g., $\Omega=1$ at $\omega=6$, there are five pendular states bound by the cosine potential, two of which have negative energies with respect to the correlating field-free states. Contrary to the first-order Zeeman effect, the energy dependence of pendular states is nonlinear in $\omega$ and for the negatively bound states it decreases with increasing field strength. The sign of the magnetic dipole moment is determined by the sign of the projection $\Omega$; consequently, for given nonzero $M, \Omega$, and $\omega$, there are just $2^{2}$ sign combinations giving rise to a quadruplet of states consisting of two isoenergetic doublets. Within each doublet the states differ by the sign of $\Omega$ and hence $\omega$ and are equally populated in an equilibrium ensemble. For either $\boldsymbol{M}$ or $\boldsymbol{\Omega}$ equal to zero there is just a doublet of isoenergetic states. We refer to this scheme as the " $M \Omega$ rule." The body-fixed magnetic dipole moment can be nonzero even for $\Omega=0$, due to the anomalous electron spin such as occurs within a Hund's case (a) ${ }^{3} \Pi_{0}$ manifold [9]; the corresponding pendular states would then coincide with those of a linear polar ${ }^{1} \Sigma$ molecule in an electric field.

For the $X$ and $A$ electronic states of $\mathrm{ICl}$ we have respectively $\Omega^{\prime \prime}=0, \mu^{\prime \prime}=0$, and $\Omega^{\prime}=1, \mu^{\prime}=1 \mu_{B}$. Consequently, the pendular hybridization only occurs for the $A$ state, $\left|\tilde{J}^{\prime}, \Omega^{\prime}, M^{\prime} ; \omega^{\prime}\right\rangle$, while within the $X$ state manifold there are just field-free rotor states, $\left|\tilde{J}^{\prime \prime}, 0, M^{\prime \prime} ; \omega^{\prime \prime}=0\right\rangle$ $=\left|J^{\prime \prime}, 0, M^{\prime \prime}\right\rangle$. For radiative transitions between a pair of such states, $\left|\tilde{J}^{\prime}, \Omega^{\prime}, M^{\prime} ; \omega^{\prime}\right\rangle-\left|J^{\prime \prime}, \Omega^{\prime \prime}, M^{\prime \prime}\right\rangle$, the line strength factor $[8,14]$ is given by 
TABLE I. Parameters for calculation of $\mathrm{I}^{35} \mathrm{Cl}$ spectra.

\begin{tabular}{lccc}
\hline & $X, v^{\prime \prime}=0$ & $A, v^{\prime}=19$ & Reference \\
\hline \hline Rotational constant & $B^{\prime \prime}=0.113891 \mathrm{~cm}^{-1}$ & $B^{\prime}=0.062510 \mathrm{~cm}^{-1}$ & [15-17] \\
Magnetic dipole moment & $\mu^{\prime \prime}=0$ & & \\
Interaction parameter & $\omega=0.4668 \mu_{B} \mathcal{H}(\mathrm{T}) / B\left(\mathrm{~cm}^{-1}\right)$ & & \\
\hline \hline
\end{tabular}

$$
\begin{aligned}
s= & \left(2 J^{\prime \prime}+1\right) \sum_{J^{\prime}, q, Q} a_{J^{2} \Omega^{\prime} M^{\prime}}\left(\omega^{\prime}\right)\left(2 J^{\prime}+1\right) \\
& \times\left(\begin{array}{ccc}
J^{\prime \prime} & 1 & J^{\prime} \\
-M^{\prime \prime} & Q & M^{\prime}
\end{array}\right)^{2}\left(\begin{array}{ccc}
J^{\prime \prime} & 1 & J^{\prime} \\
-\Omega^{\prime \prime} & q & \Omega^{\prime}
\end{array}\right)^{2} .
\end{aligned}
$$

Here $Q=0, \pm 1$ designates the spherical components in the space-fixed frame of the electric field vector $e_{Q}$ of the radiation and $q=0, \pm 1$ those in the body-fixed frame of the transition dipole operator $\mu_{q}$ for the molecule; these are taken as unit vectors, $\left|e_{Q}\right|=\left|\mu_{q}\right|=1$. For the experimental excitation or detection geometry of Fig. 1 and laser polarization parallel and perpendicular to the $z$ axis, the only nonzero components of the photon angular momentum occur respectively for $Q=0$ and for $Q= \pm 1$. Furthermore, in transitions between the $A$ and $X$ states of $\mathrm{ICl}$ there is $\Omega^{\prime}= \pm 1$ and $\Omega^{\prime \prime}=0$, corresponding to a perpendicular transition dipole moment with nonzero components for $q= \pm 1$. Hence the $3-J$ symbols in Eq. (3) specify that the allowed transitions have $\Delta M=0$ (parallel polarization) or $\Delta M= \pm 1$ (perpendicular polarization) and $\Delta \Omega= \pm 1$. However, the $a_{J^{\prime} \Omega^{\prime} M^{\prime}}$ coefficients introduced by the field-induced hybridization of $J$ states give rise to nonzero transition probabilities between states that differ by more than unity in their nominal $\tilde{J}$ values. Although the $3-J$ symbols vanish unless $\Delta J=0$ or \pm 1 , the sum over the hybrid components links states more distant in nominal $\tilde{J}$, and increasingly so as the parameter $|\omega|$ increases. This enriches the spectra with many transitions that would be forbidden in the absence of the hybridizing field. The parity selection rule is also lifted by the hybridization; however, this does not introduce any new transitions involving states with $\Omega \neq 0$ as their field-free parity is indefinite [11].

The stick spectra included in Fig. 2 were calculated from this theory. The eigenvalues and expansion coefficients for the eigenfunctions for the $A$ state of $\mathrm{ICl}$ were obtained from Eq. (1), while for the $X$ state we used free-rotor eigenproperties. The molecular parameters employed are given in Table I. For all transitions occurring in the observed frequency range, the line strengths, $s$, were evaluated from Eq. (3). The initial populations, $w J^{\prime \prime}$, were derived from the field-free spectra; they were found to correspond to a rotational temperature of about $10 \mathrm{~K}$. The stick spectra show the calculated energies, $\left(E^{\prime}-E^{\prime \prime}\right) / B$, and intensities, $I=w s$, of the transitions between the field-free rotor states (from within the $X, v^{\prime \prime}=0$ manifold) and the pendular states (from within the $A$, $v^{\prime}=19$ manifold). The band origin [15], $\tilde{v}_{0}=16652.790$ $\mathrm{cm}^{-1}$, corresponds to our abscissa origin at $\left(E^{\prime}-E^{\prime \prime}\right)$ / $B^{\prime \prime}=0$. The hyperfine structure, which is predominantly due to the quadrupole moment and spin of the iodine nucleus [15-17], has been omitted in this analysis; nevertheless, the assignment of the peak groups or broadened lines to the calculated transitions is unequivocal. The stick spectra are sorted into bands labeled by $\tilde{J}^{\prime}-J^{\prime \prime}$; these involve respectively even and odd numbers of transitions for $\Delta M= \pm 1$ and $\Delta M=0$. This is not apparent in all of the bands since some of the transitions overlap. The $\Delta M$ transitions with $\Delta \Omega=+1$ and $\Delta \Omega=-1$ are not coincident in the spectra. When the field is present $(\omega \neq 0)$, states with the same $\tilde{J}$ and $M$ but $\Omega= \pm 1$ differ in both energy and wave function, according to the $M \Omega$ rule. The corresponding pair of transitions,

$$
\left|\tilde{J}^{\prime}, 1, M^{\prime} ; \omega^{\prime}\right\rangle \leftarrow\left|J^{\prime \prime}, 0, M^{\prime \prime}\right\rangle
$$

and

$$
\left|\tilde{J}^{\prime},-1, M^{\prime} ; \omega^{\prime}\right\rangle \leftarrow\left|J^{\prime \prime}, 0, M^{\prime \prime}\right\rangle,
$$

occur at different frequencies and with different probabilities. At the same time, the $M \Omega$ rule ensures that the transitions of Eq. (4) each have an indistinguishable twin: for (4a) an equivalent transition is $\left|\tilde{J}^{\prime},-1,-M^{\prime} ; \omega^{\prime}\right\rangle$ $\leftarrow\left|J^{\prime \prime}, 0,-M^{\prime \prime}\right\rangle$; for (4b) it is $\left|\tilde{J}^{\prime},+1,-M^{\prime} ; \omega^{\prime}\right\rangle \leftarrow \mid J^{\prime \prime}, 0$, $\left.-M^{\prime \prime}\right\rangle$.

Table II illustrates typical pendular properties for two examples. These properties include a violation (here $\Delta \tilde{J}=2)$ of the usual field-free selection rule; the extent of alignment (specified by $|\langle\cos \theta\rangle|$ ), and the energy shift (given in units of the ground-state rotational constant). Note that individual molecules are oriented, with the sense depending on the sign of the magnetic dipole moment $\mu$, but the ensemble as a whole is only aligned [9]. This is a consequence of the situation summarized in the $M \Omega$ rule described above. Thus, molecules with positive and negative $\mu$ cannot be separated by state selection using a Stern-Gerlach field because the deflection is proportional to $\mu\langle\cos \theta\rangle$, which is positive definite. Optical tran-

TABLE II. Properties of observed specimen pendular states.

\begin{tabular}{cccc}
\hline & & \\
Transition & & & \\
$J^{\prime \prime}, \Omega^{\prime \prime}, M^{\prime \prime} \rightarrow \tilde{J}^{\prime}, \Omega^{\prime}, M^{\prime}$ & $\Delta \tilde{J}$ & $\langle\cos \theta\rangle^{\prime}$ & $\left(E_{J^{\prime}, \Omega^{\prime}, M^{\prime}}-E_{\tilde{J}^{\prime}, \Omega^{\prime}, M^{\prime}}\right) / B^{\prime \prime}$ \\
\hline $0,0,0 \rightarrow 2,0, \pm 1$ & 2 & 0.008497 & 0.08446 \\
$1,0,0 \rightarrow 3, \pm 1,1$ & 2 & -0.19388 & 0.43365 \\
\hline \hline
\end{tabular}


sitions between states with different signs of $\Omega$ are prohibited for $|\Omega| \geq 1$, since they would require $|\Delta \Omega| \geq 1$. Such transitions are also usually forbidden for $|\Omega|=\frac{1}{2}$ $=S_{z}$ by the spin selection rule, although this is sometimes relaxed. Therefore, ordinarily pairs of $\pm \Omega$ states behave like racemic mixtures of isomers, not inconvertible by electric dipole radiation or separable by external fields.

We also probed the $B^{3} \Pi_{0}$ states of $\mathrm{ICl}$ and $\mathrm{I}_{2}$ under the same experimental conditions, but observed no discernible field-induced spectral changes. In the case of $I_{2}$, the spectra were recorded at a resolution of $10 \mathrm{MHz}$, which confirmed the lack of any shifts or broadenings of the quadrupole hyperfine structure with magnetic field. The absence of a body-fixed magnetic moment indicates that Hund's case $(a)$ coupling does not contribute significantly. This is particularly surprising for $\mathrm{ICl}$, as the $B$ state has been considered [17] as intermediate between Hund's cases $(a)$ and $(c)$.

A.S. thanks the Alexander von Humboldt Foundation for a Feodor-Lynen Fellowship. We are grateful to Richard Anderson, Joseph Ciampi, Louis DeFeo, and David Osier (Harvard Instrumentation Shop) for prompt and skillful machining work, and Jerry Connors (Harvard University) for his untiring technical support. A research grant from the National Science Foundation is gratefully acknowledged.

[1] H. J. Loesch and A. Remscheid, J. Chem. Phys. 94, 4779 (1990); 95, 8194 (1991).

[2] B. Friedrich and D. R. Herschbach, Z. Phys. D 18, 153
(1991); B. Friedrich, D. Pullman, and D. R. Herschbach, J. Phys. Chem. 95, 8118 (1991).

[3] F. Friedrich and D. R. Herschbach, Nature (London) 353, 412 (1991).

[4] P. Block, E. Bohac, and R. E. Miller, Phys. Rev. Lett. 68, 1303 (1992).

[5] J.-M. Rost, J. Griffin, B. Friedrich, and D. R. Herschbach, Phys. Rev. Lett. 68, 1299 (1992).

[6] H. J. Loesch and J. Möller, J. Chem. Phys. 97, 9016 (1992); 97, 2158 (1993).

[7] B. Friedrich, H.-G. Rubahn, and N. Sathyamurthy, Phys. Rev. Lett. 69, 2487 (1992).

[8] B. Friedrich, D. R. Herschbach, J.-M. Rost, H.-G. Rubahn, M. Renger, and M. Verbeek, J. Chem. Soc. Faraday Trans. 89, 1539 (1993).

[9] B. Friedrich and D. R. Herschbach, Z. Phys. D 24, 25 (1992).

[10] R. B. Bernstein, D. R. Herschbach, and R. D. Levine, J. Phys. Chem. 91, 5365 (1987), and references cited therein; P. R. Brooks, Science 193, 11 (1976); S. Stolte, Nature (London) 353, 391 (1991).

[11] B. Friedrich, Chem. Phys. (to be published).

[12] A. Slenczka, B. Friedrich, and D. R. Herschbach, Rev. Sci. Instrum. (to be published).

[13] S. Gerstenkorn and P. Luc, Atlas du spectre d'absorption de la molecule du iode (CNRS, Paris, 1978).

[14] R. N. Zare, Angular Momentum (Wiley, New York, 1988).

[15] S. G. Hansen, J. D. Thompson, C. M. Western, and B. J. Howard, Mol. Phys. 49, 1217 (1983).

[16] J. R. Johnson, T. J. Slotterback, D. W. Pratt, K. C. Janda, and C. M. Western, J. Phys. Chem. 94, 5661 (1990).

[17] C. M. Western, T. J. Slotterback, J. R. Johnson, D. W. Pratt, and K. C. Janda, J. Chem. Phys. 98, 1826 (1993). 